\title{
ILS Polar Motion Results at Interannual Time Scales
}

\author{
Z. X. Li \\ Shanghai Astronomical Observatory, Chinese Academy of Sciences, 80 \\ Nandan Road, Shanghai 200030, China, e-mail: lzx@center.shao.ac.cn \\ P. Pâquet \\ Royal Observatory of Belgium, Av. Circulaire 3, 1180 Brussels, \\ Belgium, e-mail: Paul.Paquet@ksb-orb.oma.be
}

\begin{abstract}
From a comparison between the ILS interannual polar motion and that calculated from the ILS latitude data but with different combinations of the ILS stations, the ILS polar motion has been studied at interannual time scales. It has been concluded that the interannual polar motion reduced from the ILS observations is influenced by an unknown phenomenon of the Earth; the obtained polar motion is reflecting, at interannual time scales, the real motion of the pole at certain epochs only.
\end{abstract}

\section{Polar motion results at interannual time scales from ILS observa- tions.}

\subsection{ILS polar motion at interannual time scales}

After filtering using a filter with a bandpass of $1.8 \mathrm{yr}-24 \mathrm{yr}$, ILS polar motion (PM) (Yumi and Yokoyama, 1980) at interannual time scales (ITS) has been obtained. The 1947-1977 part of the ILS interannual PM (see Figure 1) is selected to be studied here because all the 5 ILS stations (Mizusawa, Kitab, Carloforte, Gaithersburg and Ukiah) were operational during this period. This result will serve as a reference in the study. The FFT is shown in Figure 2.

\subsection{Polar motion at interannual time scales for different ILS station combinations}

Beside the ordinary way of filtering the calculated polar motion $\mathrm{x}$ and $\mathrm{y}$, another way of calculating the interannual PM has been used in this study. Each of the 5 ILS latitude series has first been filtered by the bandpass filter, from which the $\mathrm{x}$ and $\mathrm{y}$ are computed afterwards. This way of calculating the interannual PM has been performed in the present study with a combination of the number of stations different from that one including the 5 ILS stations together. A comparison of the two ways of calculation shows no significant difference.

For 1947-1977, PM at ITS has also been computed by the filtered ILS observations but with different ILS station combinations as the following ( $\mathrm{M}=$ Mizusawa, $\mathrm{K}=\mathrm{Kitab}, \mathrm{C}=$ Carloforte, $\mathrm{G}=$ Gaithersburg, $\mathrm{U}=$ Ukiah): 


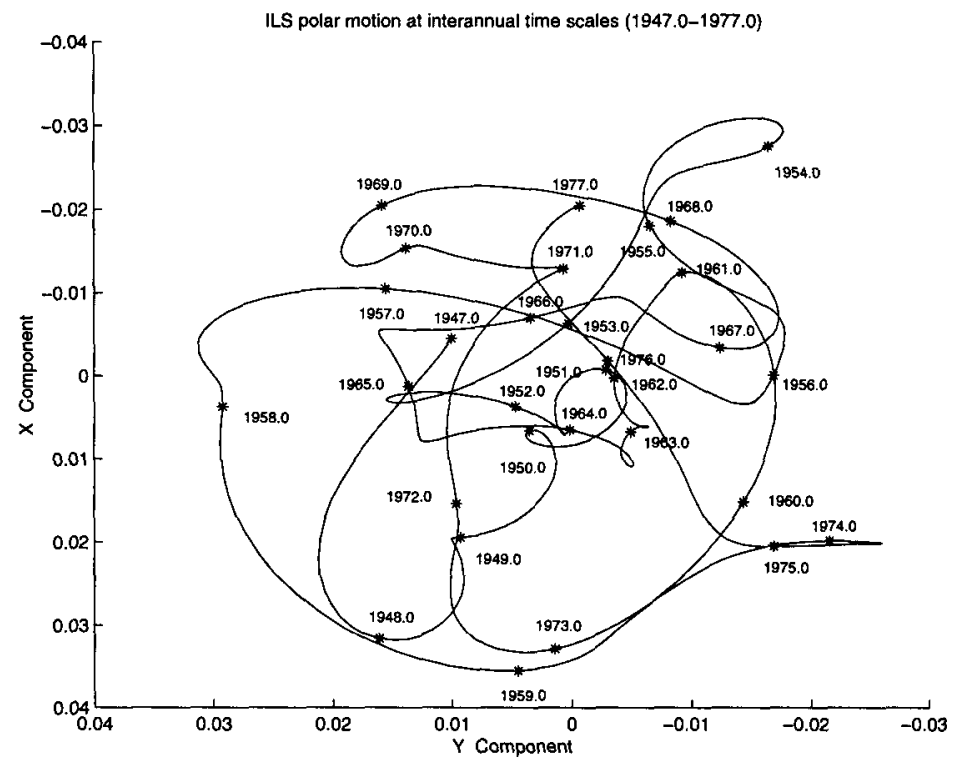

Figure 1. ILS polar motion at interannual time scales as deduced from the 5 ILS stations.
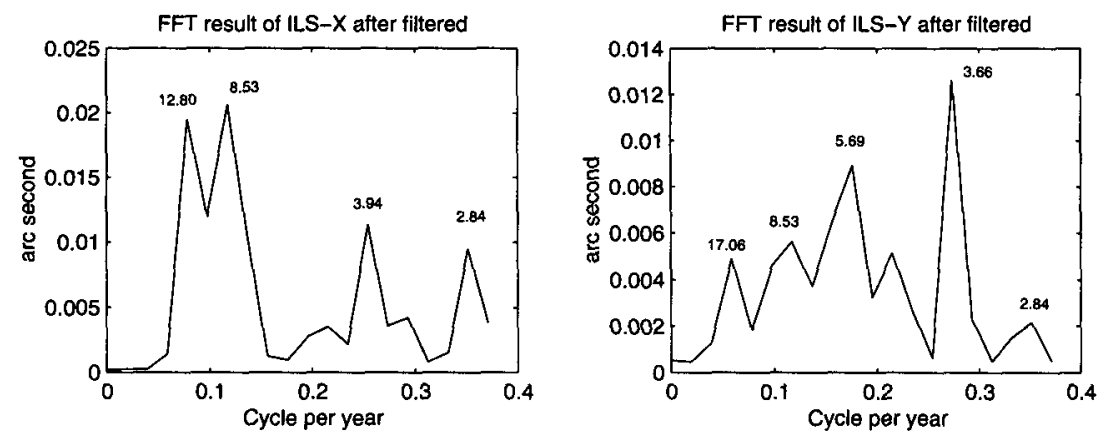

Figure 2. FFT of the ILS polar motion at interannual time scales as observed by the 5 ILS stations during the period 1947-1977. 

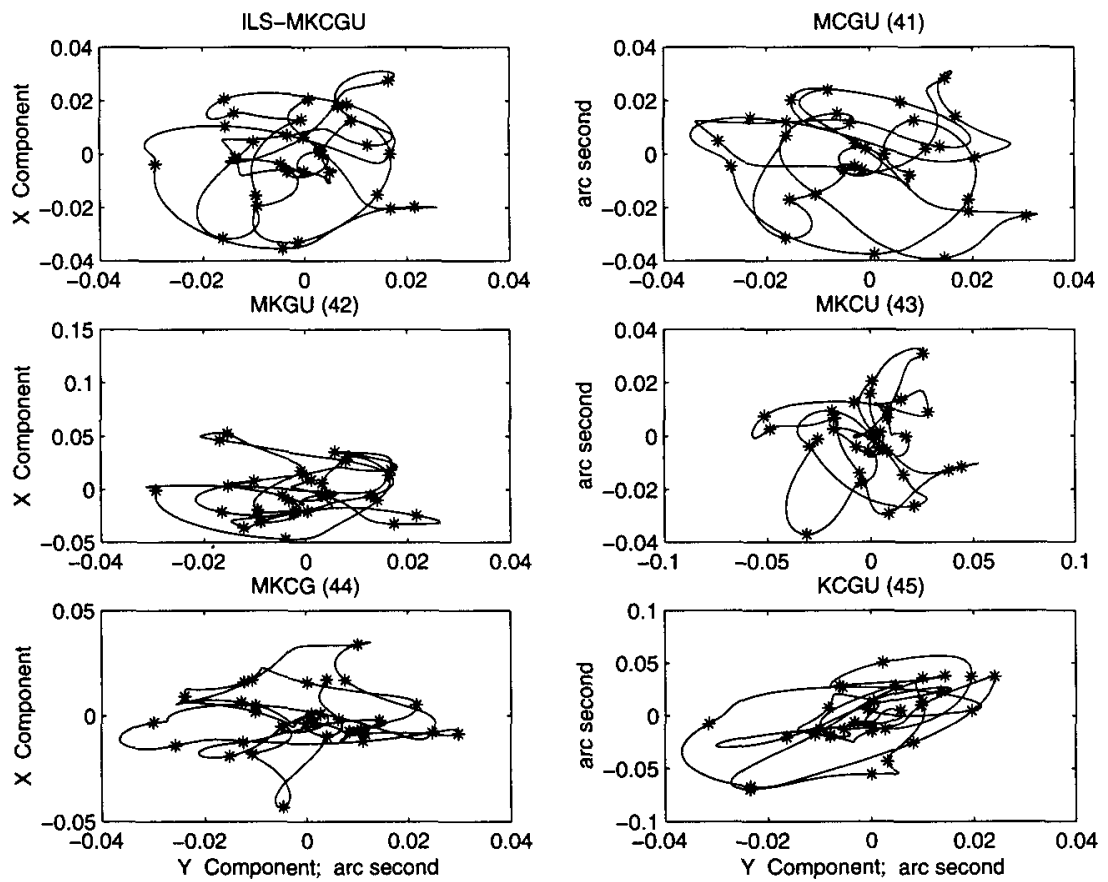

Figure 3. Interannual polar motion of the ILS and those calculated from 4 stations of the ILS (Due to a limitation of the graphic software used, all signs of the indications along the $\mathrm{x}$ and $\mathrm{y}$ axes should be reversed).

\begin{tabular}{cc}
4 Stations (Code) & 3 Stations (Code) \\
\hline M C G U (41) & M C U (31) \\
M K G U (42) & M C G $(32)$ \\
M K C U (43) & K G U (33) \\
M K C G (44) & \\
K C G U (45) &
\end{tabular}

The results are shown in Figure 3 and Figure 4.

\subsection{Comparison of PM results of different ILS station combinations at ITS}

Figure 5 shows the differences of $\mathrm{x}$ and $\mathrm{y}$ between the interannual PM of the ILS reference solution and those of the different combinations of the stations. In the top and bottom figures of Fig. 5, on each, one solution is not represented; it 

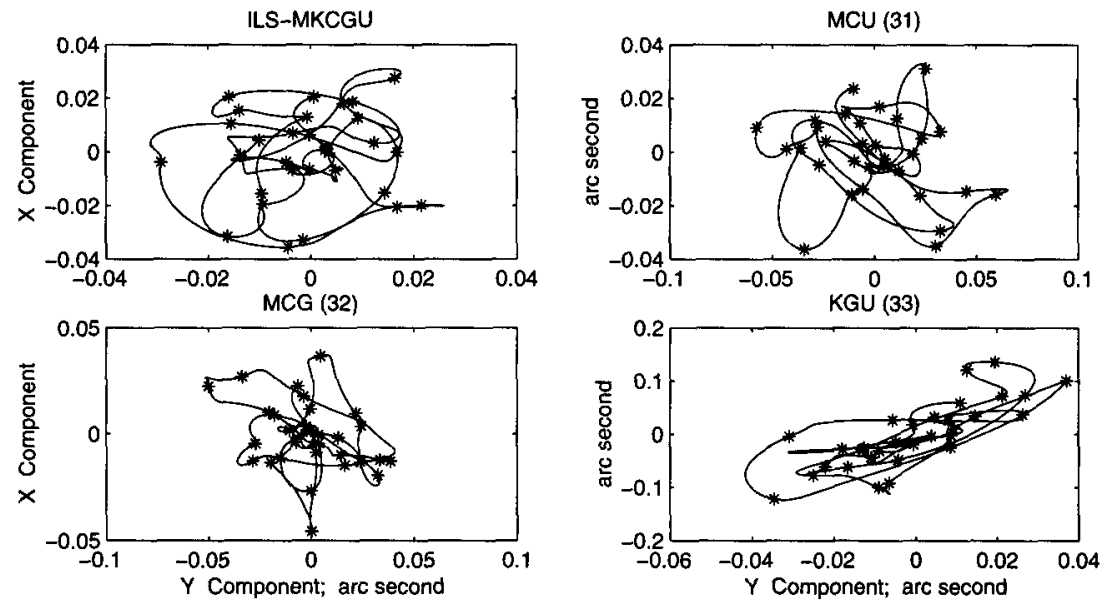

Figure 4. Interannual polar motion of the ILS and those calculated from 3 stations of the ILS (Due to a limitation of the graphic software used, all signs of the indications along the $\mathrm{x}$ and $\mathrm{y}$ axes should be reversed).

corresponds to a solution in which Carloforte (above) or Kitab (bottom) is not included in the combination of 4 stations. The reason is that the observations of these stations are of significant importance due to their strong weight in $\mathrm{x}$ and $\mathrm{y}$, respectively; indeed Carloforte is close to the $\mathrm{x}$ axis while Kitab is close to the y axis. However even if these solutions, without Carloforte or Kitab, are less precise, they do not argue against the conclusions of this paper.

As it can be seen from these figures the differences, from one combination of stations to another one, exhibit errors which change with time and whose magnitude reflects systematic and random errors of the solution. But it is remarkable to point out that, for any combination of stations, the differences with respect to the reference solution are converging near zero at the same epochs, both for $\mathrm{x}$ and $\mathrm{y}$. The epochs of convergence of all solutions are 1950, 1958, $1960,1962.5,1967.5,1972$, and 1976.

\section{Discussion}

1. PM results are affected by the existence of unavoidable errors in the observations. But why do all the observational errors of the 5 ILS stations diminish at the same time thus making the differences of any combination of stations almost disappear in the figures? It appears that the remaining "observational errors" of the 5 ILS stations are probably the "systematic errors" at ITS, while the level of the random errors is comparatively low. 

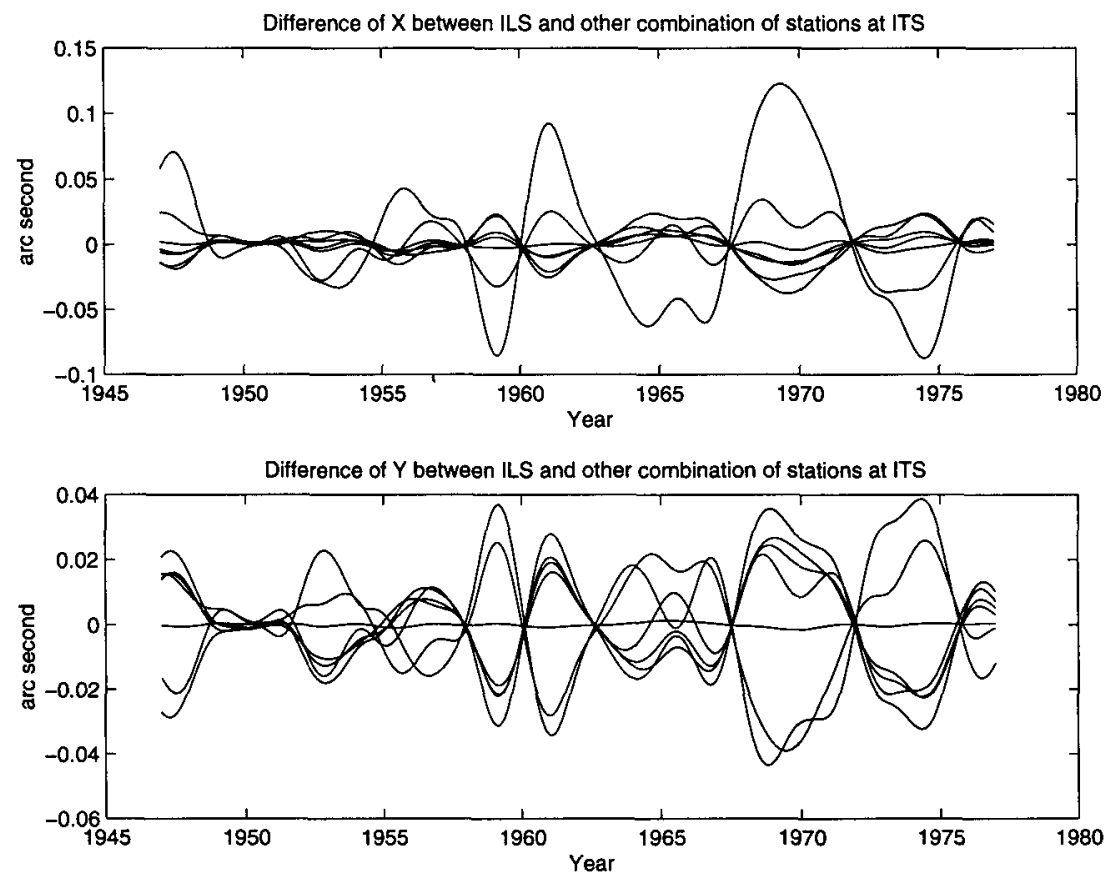

Figure 5. Differences of $\mathrm{x}$ (above) and $\mathrm{y}$ (bottom) components of the interannual polar motion computed from the 5 ILS stations and the solutions computed from 4 or 3 stations of the ILS. 
2. If there are real systematic observational errors at the 5 ILS stations, it appears in the figures that they are related to each other. They attain their maximum and minimum levels at the same time in most cases. It leads to the assumption that they are related to some unknown "global" phenomenon.

3. The PM at ITS shown in Figure 1 is significantly influenced by an unknown global phenomenon. It is not the real PM. Figure 5 shows that the effect of the global phenomenon vanishes at certain epochs: 1950, 1958, 1960, 1962.5, 1967.5, 1972 and 1976.

\section{Possible explanation and remaining questions}

1. The comparisons done at Beijing, Yunnan and Jozefoslaw between the gravimetric and astronomical techniques demonstrated the existence of vertical/plumb-line variations at ITS; the amplitudes being of the order of 0.02" ( $\mathrm{Li}, 1998)$. Is it possible that there are also similar variations of the vertical at the ILS five stations?

2. Since the unknown behavior at the ILS stations are related together as a global phenomenon, is it possible to imagine that the interannual PM in the case of ILS result is influenced by the global deformation of the geoid?

\section{Conclusions}

1. The interannual polar motion found in the ILS results is influenced by an unknown phenomenon of the Earth; it could be the variations of the local vertical at the five ILS stations. The PM at ITS reflects the real interannual PM at certain epochs only;

2. There is still interesting information in the past ILS results to be studied, particularly those related to global changes of physical parameters of the Earth; the time variations of the geoid could be a candidate.

\section{References}

Li, Z.X. 1998, Astron. Astrophys. Suppl. Ser. 129, 353-355.

Yumi, S. and Yokoyama, K. 1980, Results of the International Latitude Service in a homogeneous system, 1899.9-1979.0, Central Bureau of the International Polar Motion Service. 